\title{
Tratamento de sementes de cevada com zinco: potencial fisiológico e produtividade de sementes
}

\section{Barley seed treatment with zinc: seed physiological quality and yield}

\author{
Lizandro Ciciliano Tavares ${ }^{1}$; André Pich Brunes ${ }^{1}$; Cassyo de Araújo Rufino ${ }^{1}$; \\ Daniel Ândrei Robe Fonseca ${ }^{1}$; Gizele Ingrid Gadotti2 ${ }^{2 *}$; Francisco Amaral Villela ${ }^{2}$
}

\section{Resumo}

Objetivou-se no presente trabalho avaliar o efeito do tratamento de sementes de cevada com diferentes doses de zinco na qualidade fisiológica das sementes tratadas e produzidas, bem como avaliar o rendimento de sementes. Os tratamentos consistiram em combinações de sementes de duas cultivares e seis doses de zinco, em esquema fatorial 2 X 6 (Fator A: cultivares BRS Elis e BRS Cauê; Fator B: níveis de $0 ; 1 ; 2 ; 3 ; 4$ e $5 \mathrm{~mL} \mathrm{~kg}^{-1}$ de semente), totalizando 12 tratamentos. O delineamento experimental utilizado foi o completamente casualizado, com quatro repetições. A qualidade fisiológica das sementes tratadas e produzidas foi avaliada por testes de vigor e de germinação, sendo que após a colheita avaliouse o rendimento de sementes. O zinco fornecido via tratamento de sementes de cevada até a dose de $5 \mathrm{~mL} \mathrm{~kg}^{-1}$ de semente, nas cultivares BRS Elis e Cauê, não altera a qualidade fisiológica das sementes tratadas e produzidas e proporciona incremento no número de sementes e no rendimento.

Palavras-chave: Hordeum vulgare L., germinação, qualidade fisiológica, tratamento de sementes

\begin{abstract}
The objective of this study was to evaluate treatment in barley seeds with different doses of zinc in the physiological quality of seeds produced and treated, as well as evaluate the seed yield. The treatments consisted of combinations of two cultivars and six doses of zinc, in a factorial 2 X 6 (Factor A: - cv - BRS Ellis and BRS Cauê; Factor B: levels 0, 1, 2, 3, 4 and $5 \mathrm{~mL} \mathrm{~kg}^{-1}$ seed), totaling 12 treatments. The experimental design was completely randomized with four replications. The physiological quality of seeds produced was treated and evaluated by tests of vigor and germination, and after harvest were evaluated seed yield. Zinc supplied via barley seeds up to a dose of $5 \mathrm{~mL} \mathrm{~kg}^{-1}$ seed, the BRS Cauê and Ellis, has no effect on seed germination and vigor of treated and produced, but gives significant increases in seed number and yield.
\end{abstract}

Key words: Germination, Hordeum vulgare L., physiological quality, seed treatment

\footnotetext{
1 Discentes do Curso de Doutorado, Programa de Pós-Graduação em Ciência e Tecnologia de Sementes, Universidade Federal de Pelotas, UFPel/FAEM, Pelotas, RS. E-mail: lizandro_cicilianotavares@yahoo.com.br; beldar_brunes@msn.com; cassyo. araujo@yahoo.com.br; danielfonseca30@yahoo.com.br

2 Profs. Drs., Programa de Pós-Graduação em Ciência e Tecnologia de Sementes, UFPel/FAEM, Pelotas, RS. E-mail: gizele. gadotti@ufpel.edu.br; francisco.villela@ufpel.edu.br

* Autor para correspondência
} 


\section{Introdução}

A cevada é um cereal de inverno amplamente produzido na Região Sul do Brasil, cujos seus principais estados produtores são Rio Grande do Sul e Paraná. A cevada é o quarto cereal mais semeado no mundo, ficando atrás do trigo, arroz e milho (FAOSTAT, 2013). Pode ser utilizada para alimentação animal (pastagem ou ração), consumo humano (farinha), e usos terapêuticos, e usado especialmente para a produção de malte na indústria de cerveja (URCHEI; RODRIGUES, 1994). Os principais fatores determinantes da baixa produção de aveia são a instabilidade da produção, os elevados custos de produção e os problemas de gestão, resultando em lotes de sementes de cevada fora do padrão para a produção de malte e levando a frequentes importações de matéria-prima (GRZYBOWSKI et al., 2012).

Cerca de metade das superfícies de cereais em crescimento em todo o mundo contêm baixos níveis de $\mathrm{Zn}$ disponível no solo, e as plantas que crescem em áreas de deficiência de $\mathrm{Zn}$ e sofrem tal estresse, contêm baixos níveis de Zn no grão (OZTURK et al., 2006). Também foram constatadas deficiências em vários outros estados do País. Uma alternativa para correção da deficiência de zinco é o tratamento de sementes, com base no princípio de que a reserva da semente é importante fonte de zinco para a prevenção do desenvolvimento de sintomas iniciais de deficiência na planta.

Embora a demanda das plantas por micronutrientes seja em pequenas quantidades, a falta de qualquer um deles pode resultar em reduções significativas de produtividade. Os problemas com micronutrientes podem ocorrer por excesso de adubação fosfatada, promovendo deficiências de zinco e de manganês, calagem excessiva, insolubilizando formas de zinco e de manganês, calagem em quantidade subestimada ou mal incorporada, comprometendo a disponibilidade de molibdênio e, baixos níveis de matéria orgânica no solo, induzindo às deficiências de zinco, molibdênio e cobre (SFREDO, 2008).
A atividade do zinco é efetiva para determinados processos relevantes na homeostase fisiológica e nutricional da planta, atuando como ativador ou componente estrutural de enzimas, participante da fotossíntese nas plantas $\mathrm{C}_{4}$, através da enzima carboxilase pirúvica. É necessário para a produção de triptofano, aminoácido precursor do ácido indol acético, hormônio vegetal de crescimento envolvido no metabolismo do nitrogênio e necessário para manutenção da integridade das biomembranas (MALAVOLTA, 2006).

A absorção do zinco aplicado nas sementes ocorre quase integralmente, aumentando a reserva da semente. $\mathrm{O}$ zinco transloca-se da semente para a planta, durante e após a germinação, chegando, aos 30 dias após a emergência, a 55,5\% do total na soja, a $64 \%$ no feijão e a $69 \%$ no trigo (MURAOKA, 1981). O zinco disponível na fase inicial de crescimento da cultura, principalmente no estádio vegetativo, aplicado via semente, tem a função de estimular o desenvolvimento radicular e após translocar-se via xilema para as demais partes da planta. A absorção de zinco, no tratamento de sementes, pelas raízes é mais rápida do que a absorção e translocação de zinco aplicado via foliar em plantas de trigo, segundo Marenco e Lopes (2005). Os autores observaram ainda que durante o crescimento vegetativo, o zinco foi translocado principalmente para pontos de crescimento e folhas jovens, enquanto que durante o crescimento reprodutivo, foi translocado principalmente para folhas no caule principal e para folhas acompanhadas de órgãos reprodutivos.

É consenso entre pesquisadores de que o fornecimento dos micronutrientes através do tratamento de sementes é a forma mais eficiente, em virtude das pequenas quantidades exigidas pelas plantas, melhor uniformidade de distribuição, menor custo de aplicação e também pela garantia da nutrição no estádio inicial de crescimento, em que o sistema radicular é pouco desenvolvido, afetando a aquisição do nutriente no solo (BONECCARRÉRE et al., 2004). 
Diante desse contexto, objetivou-se avaliar o efeito do tratamento de sementes de cevada com diferentes doses de zinco no potencial fisiológico das sementes tratadas e produzidas, bem como avaliar o rendimento de sementes.

\section{Material e Métodos}

O trabalho foi conduzido no Laboratório Didático de Análise de Sementes LDAS e em casa de vegetação, da Faculdade de Agronomia Eliseu Maciel, da Universidade Federal de Pelotas.

Os tratamentos consistiram em combinações de sementes de cevada de duas cultivares tratadas com seis doses de zinco, em esquema fatorial $2 \mathrm{X}$ 6 (Fator A: cultivares BRS Elis e BRS Cauê; Fator B: doses de $0 ; 1 ; 2 ; 3 ; 4$ e $5 \mathrm{~mL}$ de zinco $\mathrm{kg}^{-1}$ de semente), totalizando 12 tratamentos, em quatro repetições. Posterior ao tratamento, as sementes foram recobertas com polímero $\left(\right.$ Sepiret $\left.^{\circledR}\right)$ na dose de $3 \mathrm{~mL} \mathrm{~kg}^{-1}$ de semente, e água em quantidade suficiente para manter o volume de calda de $12 \mathrm{~mL}$ $\mathrm{kg}^{-1}$ de semente.

As sementes foram tratadas na seguinte ordem de aplicação: nutriente + água e polímero + água. Esses produtos foram dispostos ao fundo do saco plástico, e distribuídos uniformemente até uma altura de aproximadamente $15 \mathrm{~cm}$. A seguir, adicionou-se $0,2 \mathrm{~kg}$ de sementes no interior do saco plástico, agitando por três minutos. Na sequência, as sementes foram colocadas para secar a temperatura ambiente, durante 24 horas (NUNES, 2005).

A qualidade fisiológica das sementes tratadas foi avaliada pelos testes de: Germinação (G): realizado com quatro repetições de 50 sementes distribuídas em substrato de papel, previamente umedecido em água destilada na proporção de 2,5 vezes a massa do substrato seco, e os rolos mantidos em germinador à temperatura de $20^{\circ} \mathrm{C}$. As avaliações foram efetuadas aos sete dias após a confecção dos rolos, conforme as Regras para Análise de Sementes (BRASIL, 2009) e os resultados expressos em porcentagem de plântulas normais. Primeira contagem da germinação (PCG): avaliada aos quatro dias após a confecção dos rolos por ocasião da realização do teste de germinação. Envelhecimento acelerado (EA): realizado em caixa tipo gerbox com tela metálica. Adicionaram-se $40 \mathrm{~mL}$ de água destilada ao fundo de cada caixa e as sementes, distribuindoas uniformemente em uma única camada. Em seguida, as caixas, contendo as sementes, foram tampadas e acondicionadas em incubadora do tipo BOD, a $41{ }^{\circ} \mathrm{C}$, onde permaneceram por 48 horas. Após este período, as sementes foram submetidas ao teste de germinação e avaliadas no quarto dia. Teste de frio (TF): conduzido com quatro subamostras de 50 sementes, sendo os rolos de papel colocados em sacos plásticos, os quais foram vedados e mantidos em câmara regulada à temperatura de $10^{\circ} \mathrm{C}$ durante sete dias. Após esse período, foram transferidas para um germinador e mantidas nas mesmas condições do teste de germinação, sendo avaliadas após quatro dias (CÍCERO; VIEIRA, 1994). Emergência em campo (EC): Foram semeadas quatro repetições de 50 sementes e as avaliações realizadas aos 14 dias após a semeadura, determinando-se as porcentagens de emergência de plântulas (NAKAGAWA, 1994). As unidades experimentais foram irrigadas diariamente, mantendo-se o solo próximo à capacidade de campo.

As sementes tratadas foram semeadas em vasos com capacidade de 8 litros, em quantidade de 10 sementes por vaso, permanecendo após o desbaste 5 plantas por vaso, preenchidos com solo peneirado, coletado do horizonte A1 de um Planossolo Háplico Eutrófico Solódico (EMBRAPA, 2006), pertencente à unidade de mapeamento Pelotas. A adubação foi feita de acordo com os resultados da análise de solo e recomendações da Comissão de Química e Fertilidade do Solo (CQFS - RS/SC, 2004). Utilizaram-se nitrogênio, fósforo e potássio, nas doses de 60, 110 e $100 \mathrm{~kg} \mathrm{ha}^{-1}$, respectivamente, com aplicação 14 dias antes da semeadura e a calagem realizada trinta dias antes da semeadura aplicando-se $2,2 \mathrm{t} \mathrm{ha}^{-1}$ de calcário. O teor de 
zinco no solo foi classificado como alto (acima de $0,5 \mathrm{mg} \mathrm{dm}^{-3}$ ) de acordo com a CQFS. Após, as unidades experimentais foram irrigadas diariamente mantendo-se o solo próximo à capacidade de campo.

A colheita manual foi realizada no estádio em que 2/3 das espiguetas apresentavam coloração amareloclara ou creme, caracterizando a maturação das sementes. Após avaliaram-se as seguintes variáveis: Número de espigas (NE) e número de sementes por planta (NSP): realizado por contagem das espigas e sementes em cada unidade experimental. Rendimento de sementes por planta (RSP): foi obtido pela pesagem das sementes colhidas, sendo a umidade corrigida para $13 \%$. Peso hectolítrico (PH): realizada com quatro repetições e balança específica, com capacidade de 1 litro de sementes, sendo o resultado expresso em $\mathrm{kg} \mathrm{hL}^{-1}$. Peso de mil sementes (PMS) - foram empregadas oito repetições de 100 sementes. Para estas pesagens calculou-se a média, o desvio padrão e o coeficiente de variação. Todas as repetições apresentaram coeficiente de variação inferior a quatro, portanto, multiplicou-se a média por 10, e assim obteve-se o peso de mil sementes (BRASIL, 2009).

A qualidade das sementes produzidas foi avaliada pelos testes: primeira contagem da germinação (PCG), germinação (G), envelhecimento acelerado (EA), teste de frio (TF): descritos anteriormente. Comprimento de parte aérea e raiz (CPA e CR): a avaliação do comprimento da parte aérea e da raiz foi realizada com quatro subamostras de 20 sementes para cada tratamento. Utilizouse como substrato rolo de papel para germinação do tipo "germitest", no qual as sementes foram distribuídas em duas linhas retas longitudinais e desencontradas no terço superior do papel. Após a confecção dos rolos, os mesmos foram colocados em germinador regulado à temperatura constante de $20^{\circ} \mathrm{C}$ (NAKAGAWA, 1999). No quarto dia após a semeadura, foi avaliado o comprimento da parte aérea e da raiz, das plântulas normais, sendo cada plântula medida separadamente e, em seguida, calculado o comprimento médio parte aérea e da raiz.

Os dados foram submetidos à análise de variância, sendo realizada comparações de médias para o fator qualitativo (cultivar) através do teste de Tukey, e regressão polinomial para o fator quantitativo (Doses de zinco), todos a $5 \%$ de probabilidade de erro. Para a análise estatística foi utilizado o Sistema de Análise Estatística Winstat versão 1.0 (MACHADO; CONCEIÇÃO, 2003).

\section{Resultados e Discussão}

$\mathrm{Na}$ avaliação da qualidade fisiológica após o tratamento das sementes, não foi constatada interação entre as doses de zinco e cultivares testadas para as variáveis primeira contagem da germinação, germinação, envelhecimento acelerado, teste de frio, emergência a campo, bem como não foram observados efeitos principais (Tabela 1).

Em relação as sementes colhidas não foi constatada interação entre os fatores de tratamento para nenhuma das variáveis analisadas, havendo apenas efeito principal de doses de zinco para as variáveis envelhecimento acelerado, comprimento de parte aérea (CPA) e peso de mil sementes (PMS), e apenas efeito principal de cultivar para a variável teste de frio (Tabela 2).

$\mathrm{Na}$ Tabela 3, encontram-se os dados referentes à qualidade das sementes de cevada tratadas com zinco, avaliadas pelos testes de primeira contagem de germinação, germinação, envelhecimento acelerado, teste de frio e emergência em campo. Não se verificou efeito significativo dos fatores cultivar e dose e da interação. Observa-se que as cultivares de cevada BRS Elis e Cauê não diferiram ao serem submetidas às doses crescentes de zinco via tratamento de sementes. Tais resultados estão de acordo com os obtidos por Funguetto et al. (2010), que analisando o recobrimento de sementes em arroz com fontes de zinco, fungicida e polímero, não verificaram alteração na germinação. 
Resultados semelhantes também foram obtidos por Rozane et al. (2008) que utilizaram doses de zinco em sementes de arroz (1,0; 2,0; 4,0 e 8,0 $\mathrm{g} \mathrm{Zn} \mathrm{kg}^{-1}$ sementes), tendo como fontes sulfato e óxido de zinco comparados com a testemunha sem zinco. No entanto, em sementes de sorgo tratadas com zinco, Yagi et al. (2006) constataram diminuição da percentagem de germinação.

Tabela 1. Análise de variância (ANOVA) dos fatores dose de zinco e cultivares em sementes de cevada tratadas com doses de zinco, para as variáveis primeira contagem da germinação (PCG), germinação (G), envelhecimento acelerado (EA), teste de frio (TF) e emergência a campo (EC), em sementes de cevada tratadas com doses de zinco.

\begin{tabular}{lccccc}
\hline Fatores de tratamento & PCG & G & EA & TF & EC \\
\hline Dose & 0,977 & 0,452 & 0,327 & 0,943 & 0,326 \\
Cultivar & 0,994 & 0,946 & 0,587 & 0,656 & 0,307 \\
Dose*Cultivar & 0,996 & 0,935 & 0,999 & 0,991 & 0,999 \\
\hline
\end{tabular}

Fonte: Elaboração dos autores.

Tabela 2. Análise de variância (ANOVA) dos fatores dose de zinco e cultivares em sementes de cevada colhidas, para as variáveis primeira contagem da germinação (PCG), germinação $(\mathrm{G})$, envelhecimento acelerado (EA), teste de frio (TF) comprimento da parte aérea (CPA), comprimento de raiz (CR), peso hectolítrico (PH), e peso de mil sementes (PMS).

\begin{tabular}{lcccc}
\hline Fatores de tratamento & PCG & G & EA & TF \\
\hline Dose & 0,801 & 0,801 & 0,0001 & 0,541 \\
Cultivar & 0,849 & 0,849 & 0,901 & 0,032 \\
Dose*Cultivar & 0,106 & 0,106 & 0,153 & 0,098 \\
\hline & CPA & CR & PH & PMS \\
\hline Dose & 0,000 & 0,045 & 0,253 & 0,000 \\
Cultivar & 0,707 & 0,176 & 0,224 & 0,060 \\
Dose*Cultivar & 0,377 & 0,089 & 0,072 & 0,860 \\
\hline
\end{tabular}

Fonte: Elaboração dos autores.

Tabela 3. Primeira contagem da germinação (PCG), germinação (G), envelhecimento acelerado (EA), teste de frio (TF) e emergência em campo de plântulas (EC) de sementes de cevada tratadas com doses de zinco. Capão do Leão, RS, 2010.

\begin{tabular}{|c|c|c|c|c|c|c|c|c|c|c|}
\hline \multirow{3}{*}{$\begin{array}{c}\text { Dose } \mathrm{mL} \\
\mathrm{kg}^{-1}\end{array}$} & \multicolumn{10}{|c|}{ Cultivar } \\
\hline & Ellis & Cauê & Ellis & Cauê & Ellis & Cauê & Ellis & Cauê & Ellis & Cauê \\
\hline & \multicolumn{2}{|c|}{ PCG $(\%)$} & \multicolumn{2}{|c|}{$\mathrm{G}(\%)$} & \multicolumn{2}{|c|}{ EA $(\%)$} & \multicolumn{2}{|c|}{ TF $(\%)$} & \multicolumn{2}{|c|}{ EC $(\%)$} \\
\hline 0 & 81 & 80 & 89 & 90 & 81 & 80 & 79 & 79 & 78 & 79 \\
\hline 1 & 81 & 81 & 91 & 91 & 82 & 81 & 80 & 79 & 81 & 83 \\
\hline 2 & 80 & 81 & 91 & 91 & 82 & 81 & 80 & 81 & 81 & 84 \\
\hline 3 & 81 & 81 & 91 & 91 & 83 & 82 & 81 & 81 & 82 & 84 \\
\hline 4 & 80 & 80 & 92 & 90 & 84 & 82 & 82 & 81 & 83 & 85 \\
\hline 5 & 81 & 80 & 93 & 90 & 85 & 84 & 81 & 82 & 84 & 85 \\
\hline Média & $80 \mathrm{~A}$ & $80 \mathrm{~A}$ & $91 \mathrm{~A}$ & $91 \mathrm{~A}$ & $83 \mathrm{~A}$ & $81 \mathrm{~A}$ & $80 \mathrm{~A}$ & $80 \mathrm{~A}$ & $82 \mathrm{~A}$ & $83 \mathrm{~A}$ \\
\hline C.V. $(\%)$ & \multicolumn{2}{|c|}{6.1} & \multicolumn{2}{|c|}{4.2} & \multicolumn{2}{|c|}{5.1} & \multicolumn{2}{|c|}{5.0} & \multicolumn{2}{|c|}{6.8} \\
\hline
\end{tabular}

*Médias seguidas pela mesma letra maiúscula na linha, em cada variável resposta, não diferem pelo teste de Tukey a 5\% de probabilidade.

Fonte: Elaboração dos autores. 
Os dados apresentados na Tabela 4 referem-se à qualidade fisiológica das sementes produzidas oriundas do tratamento das sementes com doses de zinco. Observa-se que a cultivar BRS Elis apresentou maior desempenho que a cultivar BRS Cauê no comprimento da parte aérea de plântulas. Já a cultivar BRS Cauê apresentou desempenho superior ao da cultivar BRS Elis no teste de envelhecimento acelerado e no comprimento de raiz de plântulas.
$\mathrm{Na}$ Tabela 5 encontram-se os dados referentes ao número de espigas, número de sementes por espiga, rendimento de sementes e peso hectolítrico de sementes de cevada originadas do tratamento com doses de zinco. Não ocorreu interação entre os fatores, tampouco entre os efeitos principais de cultivares e doses de zinco. Observa-se que tanto a cultivar BRS Elis como a cultivar BRS Cauê, obtiveram comportamento semelhante ao serem submetidas a doses de zinco via tratamento das sementes.

Tabela 4. Primeira contagem da germinação (PCG), germinação (G), envelhecimento acelerado (EA), teste de frio (TF), comprimento de parte aérea (CPA), comprimento de raiz (CR) de sementes de cevada produzidas, provenientes do tratamento com doses de zinco. Capão do Leão, RS, 2010.

\begin{tabular}{|c|c|c|c|c|c|c|c|c|c|c|c|c|}
\hline \multirow{3}{*}{$\begin{array}{c}\text { Dose } \mathrm{mL} \\
\mathrm{kg}^{-1}\end{array}$} & \multicolumn{12}{|c|}{ Cultivar } \\
\hline & Ellis & Cauê & Ellis & Cauê & Ellis & Cauê & Ellis & Cauê & Ellis & Cauê & Ellis & Cauê \\
\hline & \multicolumn{2}{|c|}{ PCG (\%) } & \multicolumn{2}{|c|}{ G (\%) } & \multicolumn{2}{|c|}{ EA $(\%)$} & \multicolumn{2}{|c|}{ TF $(\%)$} & \multicolumn{2}{|c|}{$\mathrm{CPA}(\mathrm{cm})$} & \multicolumn{2}{|c|}{$\mathrm{CR}(\mathrm{cm})$} \\
\hline 0 & 92 & 90 & 98 & 95 & 95 & 93 & 100 & 99 & 3,8 & 3,3 & 5,5 & 5,2 \\
\hline 1 & 90 & 94 & 96 & 99 & 89 & 97 & 97 & 98 & 3,9 & 3,3 & 5,5 & 5,7 \\
\hline 2 & 92 & 94 & 96 & 98 & 93 & 96 & 99 & 99 & 3,9 & 3,4 & 5,7 & 5,6 \\
\hline 3 & 92 & 95 & 97 & 99 & 91 & 97 & 98 & 99 & 3,9 & 3,3 & 5,6 & 6,0 \\
\hline 4 & 94 & 92 & 98 & 96 & 93 & 97 & 94 & 98 & 3,9 & 3,1 & 5,5 & 6,1 \\
\hline 5 & 96 & 93 & 99 & 97 & 93 & 98 & 98 & 95 & 3,8 & 3,3 & 5,5 & 6,3 \\
\hline Média & $93 \mathrm{~A}$ & $93 \mathrm{~A}$ & $97 \mathrm{~A}$ & $97 \mathrm{~A}$ & $92 \mathrm{~B}$ & $96 \mathrm{~A}$ & $97 \mathrm{~A}$ & $98 \mathrm{~A}$ & $3,9 \mathrm{~A}$ & $3,3 \mathrm{~B}$ & $5,6 \mathrm{~B}$ & $5,8 \mathrm{~A}$ \\
\hline C.V. $(\%)$ & \multicolumn{2}{|c|}{3.0} & \multicolumn{2}{|c|}{2.9} & \multicolumn{2}{|c|}{3.7} & \multicolumn{2}{|c|}{2.3} & \multicolumn{2}{|c|}{5.5} & \multicolumn{2}{|c|}{5.6} \\
\hline
\end{tabular}

*Médias seguidas pela mesma letra maiúscula na linha, em cada variável resposta, não diferem pelo teste de Tukey a $5 \%$ de probabilidade.

Fonte: Elaboração dos autores.

Tabela 5. Número de espigas (NE), número de sementes por espiga (NSE), rendimento de sementes (R) e peso hectolítrico $(\mathrm{Ph})$ provenientes de sementes de cevada tratadas com zinco. Capão do Leão, RS, 2010.

\begin{tabular}{|c|c|c|c|c|c|c|c|c|}
\hline \multirow{3}{*}{ Dose $\mathrm{mL} \mathrm{kg}{ }^{-1}$} & \multicolumn{8}{|c|}{ Cultivar } \\
\hline & Ellis & Cauê & Ellis & Cauê & Ellis & Cauê & Ellis & Cauê \\
\hline & \multicolumn{2}{|c|}{ NE (Unidades) } & \multicolumn{2}{|c|}{ NSE (Unidades) } & \multicolumn{2}{|c|}{$\mathrm{R}\left(\mathrm{g} \mathrm{planta}^{-1}\right)$} & \multicolumn{2}{|c|}{$\mathrm{Ph}\left(\mathrm{kg} \mathrm{hl}^{-1}\right)$} \\
\hline 0 & 9 & 9 & 16 & 18 & 6,2 & 6,3 & 56,4 & 55,6 \\
\hline 1 & 9 & 9 & 18 & 20 & 6,6 & 6,6 & 56,8 & 57,8 \\
\hline 2 & 9 & 9 & 19 & 21 & 6,8 & 6,9 & 58,0 & 58,0 \\
\hline 3 & 9 & 9 & 21 & 22 & 6,9 & 7,0 & 58,0 & 58,6 \\
\hline 4 & 9 & 9 & 22 & 22 & 6,9 & 7,3 & 55,8 & 58,6 \\
\hline 5 & 9 & 10 & 23 & 24 & 7,5 & 7,6 & 57,8 & 58,4 \\
\hline Média & $9 \mathrm{~A}^{*}$ & $9 \mathrm{~A}$ & $20 \mathrm{~A}$ & $21 \mathrm{~A}$ & $6,8 \mathrm{~A}$ & $7,0 \mathrm{~A}$ & $57,1 \mathrm{~A}$ & $57,8 \mathrm{~A}$ \\
\hline C.V. $(\%)$ & \multicolumn{2}{|c|}{13,6} & \multicolumn{2}{|c|}{15,6} & \multicolumn{2}{|c|}{9,5} & \multicolumn{2}{|c|}{2,6} \\
\hline
\end{tabular}

*Médias seguidas pela mesma letra maiúscula na linha, em cada variável resposta, não diferem pelo teste de Tukey a 5\% de probabilidade.

Fonte: Elaboração dos autores. 
$\mathrm{Na}$ Figura 1 são apresentados os dados referentes ao número de sementes por planta e rendimento de sementes. Verifica-se que as duas variáveis apresentaram respostas lineares, ou seja, com o aumento de zinco ocorreu aumento no número de sementes por planta e rendimento de sementes, sendo esse incremento de 20 e $35 \%$ na dose de 5 $\mathrm{mL} \mathrm{kg}$ de semente, respectivamente, relativamente a dose zero. Muraoka (1981) justifica esses dados, pois com o incremento de zinco há o incremento de reserva da semente. Já Marenco e Lopes (2005) explicam que o zinco é translocado para folhas acompanhadas de órgãos reprodutivos. Os dados obtidos concordam com os encontrados por Funguetto et al. (2010), que obtiveram aumento linear das variáveis número de grãos por panícula e no peso de grãos por planta com a elevação das doses de zinco, apresentando incremento de $29 \%$ no número de grãos por panícula e $21 \%$ no peso de grãos por planta, na dose máxima de $0,77 \mathrm{~g}$ de zinco $\mathrm{kg}^{-1}$ de sementes, comparativamente à dose zero. Entretanto, Orioli Junior et al. (2008) não detectaram diferenças no número de espiguetas em sementes de trigo tratadas com zinco.

Figura 1. Média do número de sementes por planta (A) e rendimento de sementes (B) de cevada, cultivares BRS Elis e Cauê, provenientes do tratamento com doses de zinco via tratamento de sementes. Capão do Leão, RS, 2010.

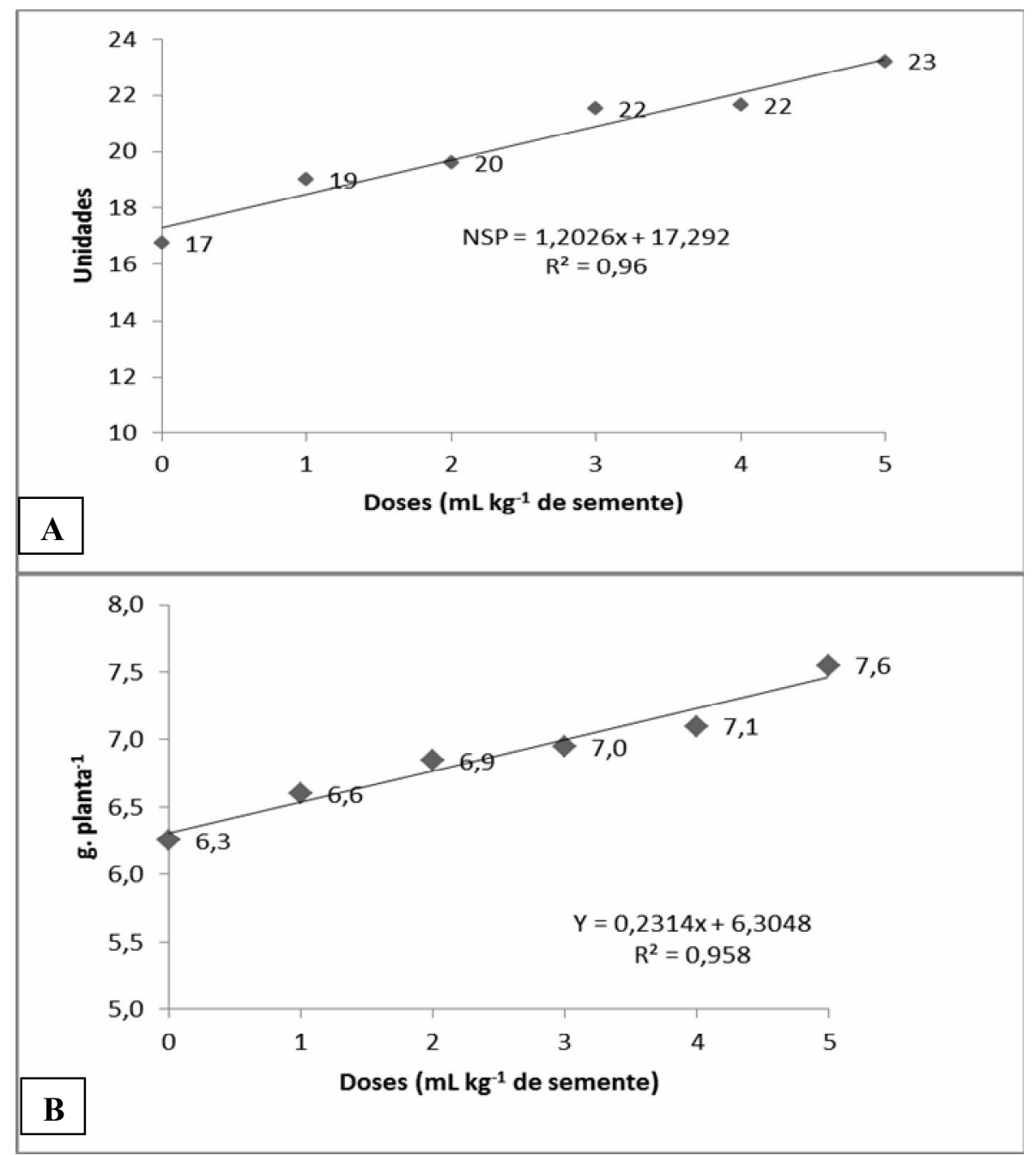

Fonte: Elaboração dos autores. 
Uma análise geral dos resultados alcançados com o tratamento de sementes de cevada, com doses crescentes de zinco permite verificar que não ocorreram alterações nos atributos de qualidade tanto após o tratamento das sementes quanto para as sementes produzidas a partir das sementes tratadas. Não obstante, as variáveis número de sementes por planta e rendimento de sementes apresentaram incrementos lineares com a elevação das doses de zinco.

\section{Conclusão}

O zinco fornecido via tratamento de sementes de cevada até a dose de $5 \mathrm{~mL} \mathrm{~kg}^{-1}$ de semente, nas cultivares BRS Elis e Cauê, não altera a qualidade fisiológica das sementes tratadas e produzidas, e incrementa o número de sementes por planta e rendimento.

\section{Referências}

BONNECARRÈRE, R. A. G.; LONDERO, F. A. A.; SANTOS, O.; SCHMIDT, D.; PILAU, F. G. MANFRON, P. A.; DOURADO NETO, D. Resposta de genótipos de arroz irrigado à aplicação de zinco. Revista Faculdade Zootecnia Veterinária e Agronomia, Uruguaiana, v. 10, n. 1, p. 214-222, 2004.

BRASIL. Ministério da Agricultura, Pecuária e Abastecimento. Regras para análise de sementes. Ministério da Agricultura, Pecuária e Abastecimento. Secretaria de Defesa Agropecuária. Brasília: Mapa/ACS, 2009. $395 \mathrm{p}$.

CÍCERO, S. M.; VIEIRA, R. D. Teste de frio. In: VIEIRA, R. D.; CARVALHO, N. M. Testes de vigor em sementes. Jaboticabal: FUNEP, 1994. p. 151-164.

COMISSÃO DE QUÍMICA E FERTILIDADE DO SOLO - CQFS - RS/SC. Manual de adubação e de calagem para os estados do Rio Grande do Sul e de Santa Catarina. 10. ed. Porto Alegre: NRS/SBCS, 2004. 400 p.

EMPRESA BRASILEIRA DE PESQUISA AGROPECUÁRIA - EMBRAPA. Centro Nacional de Pesquisa de Solos. Sistema brasileiro de classificação de solos. 2. ed. Rio de Janeiro: EMBRAPA-SPI, 2006. 306 p.
FOOD AND AGRICULTURE ORGANIZATION OF THE UNITED NATIONS - FAOSTAT. Statistical databases. Roma: FAO, 2013. Disponível em: <http:// faostat.fao.org>. Acesso em: 2 dez. 2014.

FUNGUETTO, C. I.; PINTO, J. F.; BAUDET, L.; PESKE, S. T. Desempenho de sementes de arroz irrigado recobertas com zinco. Revista Brasileira de Sementes, Brasília, v. 32, n. 2, p. 117-115, 2010.

GRZYBOWSKI, C. R. S.; OHLSON, O. C.; SILVA, R. C.; PANOBIANCO, M. Viability of barley seeds by the tetrazolium test. Revista Brasileira de Sementes, Brasília, v. 34, n. 1, p. 47-54, 2012.

MACHADO, A. A.; CONCEIÇÃO, A. R. Sistema de análise estatística para Windows. Winstat. Versão 2.0. Pelotas: UFPel, 2003.

MALAVOLTA, E. Manual de nutrição mineral de plantas. Piracicaba: Ceres, 2006. 638 p.

MARENCO, R. A.; LOPES, N. F. Fisiologia vegetal: fotossíntese, respiração, relação hídricas e nutrição mineral. Viçosa, MG: Editora UFV, 2005. 541 p.

MURAOKA, T. Solubilidade do zinco e do manganês em diversos extratores e disponibilidade desses dois micronutrientes para o feijoeiro (Phaseolus vulgaris L.) cv. Carioca. 1981. Tese (Doutorado em Agronomia) - Escola Superior de Agricultura Luiz de Queiroz, Piracicaba.

NAKAGAWA, J. Teste de vigor baseados na avaliação de plântulas. In: VIEIRA, R. D.; CARVALHO, N. M. Testes de vigor em sementes. Jaboticabal: FUNEP, 1994, p. 49-86.

Testes de vigor baseados no desempenho das plântulas. In: KRZYZANOWSKI, F. C.; VIEIRA, R. D.; FRANÇA-NETO, J. B. Vigor de sementes: conceitos e testes. Londrina: ABRATES, 1999. cap. 2, p. 9-13.

NUNES, J. C. Tratamento de semente: qualidade e fatores que podem afetar a sua performance em laboratório. Londrina: Syngenta Proteção de Cultivos, 2005. 16 p.

ORIOLI JUNIOR, V.; PRADO, R. M.; LEONEL, C. L.; CAZETTA, D. A.; DA SILVEIRA, C. M.; QUEIROZ, R. J. B.; BASTOS, J. C. H. A. G. Modos de aplicação de zinco na nutrição e na produção de massa seca de plantas de trigo. Revista de la Ciencia del Suelo y Nutrición Vegetal, Temuco, v. 8, n. 1, p. 28-36, 2008.

OZTURK, L.; YAZICI, M. A.; YUCEL, C.; TORUN, A.; CEKIC, C.; BAGCI, A.; OZKAN, H.; BRAUN, H.-J.; SAYERS, Z.; CAKMAK, I. Concentration and localization of zinc during seed development and germination in wheat. Physiologia Plantarum, v. 128, n. 1, p. 144-152, 2006. 
ROZANE, D. E. ROZANE, D. E.; PRADO, R. M.; ROMUALDO, L. M.; SIMÕES, R. R. Resposta de plântulas de arroz c. v. Soberana à aplicação de zinco via semente. Ciência e Agrotecnologia, Lavras, v. 32, n. 3, p. 847-854, 2008.

SFREDO, G. J. Soja no Brasil: calagem, adubação e nutrição mineral. Londrina: EMBRAPA Soja, set. 2008. 148 p. (Documento, 305).
URCHEI, M. A.; RODRIGUES, J. D. Efeitos de potenciais de água no solo, em diferentes estádios fenológicos da cultura da cevada (Hordeum vulgare L.). Scientia Agricola, Piracicaba, v. 53, n. 3, p. 533-540, 1994.

YAGI, R.; SIMILI, F. F.; ARAÚJO, J. C.; PRADO, R. M.; SANCHEZ, S. V.; RIBEIRO, C. E. R.; BARRETTO, V. C. M. Aplicação de zinco via sementes e seu efeito na germinação, nutrição e desenvolvimento inicial do sorgo. Pesquisa Agropecuária Brasileira, Brasília, v. 41, n. 4, p. 655-660, 2006. 
